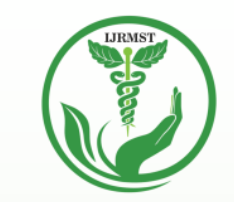

INTERNATIONAL JOURNAL OF RESEARCH IN MEDICAL SCIENCES \& TECHNOLOGY

e-ISSN:2455-5134; p-ISSN: 2455-9059

\title{
ARTIFICIAL BLOOD: AN ACCOUNT OF HBOC AND PFC APPROACHES
}

*Aditya Misra, **Vandana Thukral

*Student, St Robert Catholic High School, Toronto, Canada

**Ph D, Skillarthi, Greater Noida

Paper Received: $12^{\text {th }}$ August, 2021; Paper Accepted: $19^{\text {th }}$ October, 2021 ;

Paper Published: $22^{\text {nd }}$ October, 2021

DOI: http://doi.org/10.37648/ijrmst.vl li02.011

\section{How to cite the article:}

Aditya Misra, Vandana Thukral, Artificial Blood: An Account of HBOC and PFC Approaches, IJRMST, JulyDecember 2021, Vol 12, 121-136, DOI: http://doi.org/10.37648/ijrmst.v11i02.011

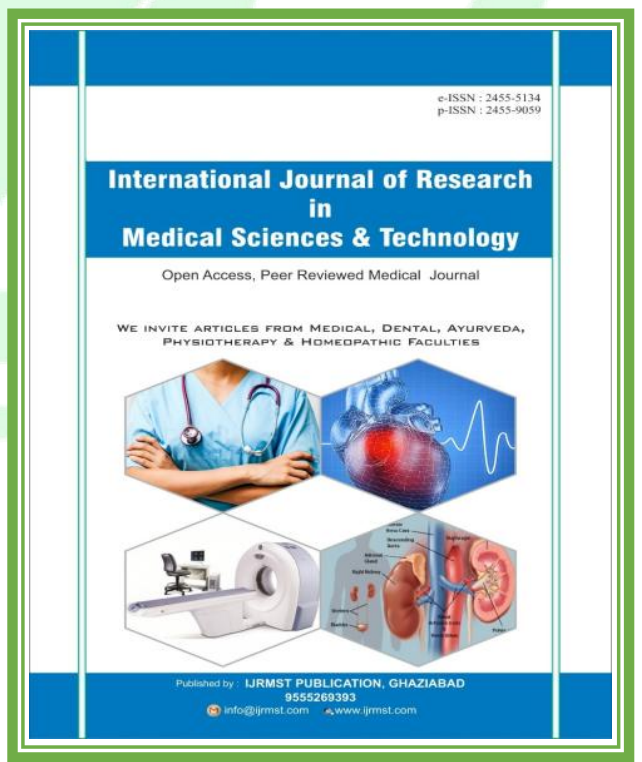




\section{ABSTRACT}

In the field of medicine, artificial blood is an innovative concept, where specially designed compounds are developed to perform the task of transport and delivery of oxygen. Hence, it can potentially replace the function of allogenic human blood transfusion.

Several molecules have been developed using several approaches. However, with continuous refinements in the past few decades, the ideal blood substitute would likely be Hemoglobin Based Oxygen Carrier. The benefits of HBOCs are tremendous, as they do not require compatibility testing or tissue matching, are non-pathogenic, have a long shelf life, and can even be stored at room temperature.

The advent of artificial blood is projected to have a remarkable impact on medical care in the future. While it will complement blood transfusion safely, it will also create a stable supply of effective products. It is likely to reduce the requirements of blood transfusions drastically in settings of surgery, trauma, or warfare.

Keywords: Anaemia; blood; erythrocytes; haemoglobin; hypersensitivity; liposomes; transfusion.

\section{INTRODUCTION}

The primary role of red blood cells (RBCs) present in the blood is to transport oxygen. It is controlled by a highly regulated mechanism, which involves a tetrameric protein called hemoglobin $(\mathrm{Hb})$. This molecule is composed of four globin molecules as two $\alpha$ - and two $\beta$-polypeptide chains. Each of these polypeptide chains contains a central iron-containing 'heme' group, each of which is capable of binding to one oxygen molecule. About 270 million Hemoglobin molecules are present in a single $\mathrm{RBC}$ and therefore they account for the tremendous oxygen carried by Red Blood Cells in lungs and tissues.

In case of severe loss of blood, also called exsanguinating hemorrhage, a sharp loss of blood RBCs occurs. Consequently, if tissue oxygen levels drop sub-optimally, it potentiates situations for morbidity and mortality. Such situations are very common; in the case of the military, surgery, and even civilian trauma. 
Under such conditions, the only way to significantly increase survival is blood transfusion. With the benefits of early action in the golden hour, a life is saved. However, the issues of limited availability, logistics, need for tissue match, short shelf-life and pathogenic contamination of biological tissue cannot be ruled out. Especially, when blood is logistically unreachable in a remote location or in a battlefield, it calls for the development of a substitute.

To resolve these issues, extensive research is being conducted towards developing artificial blood components. Blood consists of three main components, i.e., White blood cells, Red Blood Cells, and Platelets. White Blood Cells help in defense against pathogens. Red blood cells transport oxygen and carbon dioxide. Platelets and other proteins actively participating in the formation of a mesh to prevent bleeding.

Current research is designed to replace the function of Red Blood Cells, to better transport oxygen to compensate for the loss of Red Blood Cells. The use of discrete molecules to create synthetic and semi-synthetic surrogates of RBCs has been exemplified by PFCs and HBOCs.

Potential molecules to Replace RBCs could either be Perfluorochemicals or HBOCs. Perfluorochemicals or PFCs are long-chain polymers similar to Teflon. In experiments with mice, it was found that they could survive even after being immersed in fluid PFCs. Therefore, scientists used it to develop blood thinners. While trying to replace rat blood with PFCs, it made them survive for a few hours and the animal recovered fully on replacing it with their blood.

HBOCs are synthetically modified Hemoglobin molecules.

\section{HISTORICAL BACKGROUND}

About a century ago, before blood and its components were identified and blood was categorized as different blood groups, scientists investigated many solutions for their ability to substitute blood and its functions. Ranging from beer, milk, urine, plant resins, saline solutions, sheep blood, animal plasma, and animal hemoglobin, many solutions were investigated for their ability to substitute the functions of blood. The first successful blood transfusions were done in 1667 , however, unfortunately, the practice was stalled because patients who received subsequent transfusions didn't survive.

Each of the tried solutions had limited to no success to replace blood. As milk injections were already used to treat Asiatic cholera, it was also investigated. It was counter-intuitive to what scientists perceived, as milk didn't help regenerate white blood cells. 
Another potential substitute was the saline solution. In experiments on frogs, though, scientists were able to keep frogs alive if they replaced some/all of their blood with saline. However, such results were misleading as frogs can survive for a while without any blood circulation. This fact was determined later that even without any blood circulation, frogs could survive a short while. However, after much research, saline was developed as a blood volume expander.

With the development of science, blood components were identified and separated into Red Blood Cells and platelets, of which Hemoglobin was found to be the key molecule for Oxygen transport. In 1871, they identified the use of animal plasma and blood as a human blood substitute. However, scientists weren't able to procure a large volume of these fluids. Firstly, this was because they could not isolate large volumes of blood, and secondly the use from other animals could be dangerous as their blood contained many pathogenic contaminants. As the lack of technology to separate different components from another animal source was still in want, thus was an impediment.

A significant breakthrough happened whilst an attempt at Ringer's solution. This solution is composed of sodium, potassium, and calcium salts. On applying Ringer's solution on a part of a frog's heart, it was found that this solution let the heart palpitating. Therefore, it led to important findings that the reduction in blood pressure owing to blood volume loss could be restored by Ringer's solution. The addition of lactate to Ringer solution, therefore, an evolved form, began to be used as a blood volume expander. However, it was not considered a 'true' blood substitute as it couldn't replace the function of RBC.

With the extensive research efforts of Karl Landsteiner, he was able to establish blood types and thus made blood group classification a routine affair. He names these groups $\mathrm{A}, \mathrm{B}, \mathrm{O}$, and $\mathrm{AB}$.The most important contribution to the field of science thus helped match the blood type of donor and patient beforehand and making blood transfusion a safe and routine affair.

However, the blood research didn't move forward till scientists comprehensively understood the blood and its components. A gum saline solution containing galactosogluconic acid was used to extend plasma. It allowed the physicians to use less plasma by regulating concentration, $\mathrm{pH}$, and viscosity. However, it was harmful to the health of recipients. Therefore the usage declined gradually until the 1930 s. 
At the brink of World War II, the research in this area re-ignited to save the soldiers from exsanguinating and hemorrhagic shocks. Therefore, blood and blood substitutes were researched and plasma banks and blood bank systems were established.

In later years, in the years following 1966 developments, there was a competitive search to artificially synthesize blood components. Two mainly researched molecules are HBOCs and PFCs for their potential capacity to substitute urgent blood needs and functions.

The research on blood substitutes came to a standstill owing to a loss of interest in blood substitutes. In years following the Vietnam conflict, however, there was a renewed interest in the field of blood and synthetic oxygencarrying substitutes. The research therefore ensued, and was further established when in 1986, the highly transmissible HIV and hepatitis viruses were identified to be transmitted through Blood transfusions.

\section{DESIGNING OF HBOCs}

Hemoglobin carries oxygen from the lungs to the tissues in the body. Hemoglobin-based artificial blood products eliminate the step of tissue typing as they are not contained in a membrane. However, raw hemoglobin can be toxic, because it could release breakdown peptides or products which could be immunogenic/ or otherwise toxic. There are two ways to circumvent this issue. The first, linking, and second, encapsulation. Linking can be done using various approaches of cross-linking the polymeric forms. In the second approach, the encapsulated forms of $\mathrm{Hb}$ are being developed by using artificial membranes of liposomes. However, other novel approaches are also being developed and studied. Therefore, when transfuse-able blood or RBCs are not available, Hb-based oxygen carriers (HBOCs) can potentially provide therapeutic oxygenation.

Despite the rigorous research, the concept is still in preclinical and clinical evaluation. Thus a clinical approval in the USA is still deferred for human use. With several new designs and molecules with unique properties being independently studied, a few of these molecules will find their way into medicine in the subsequent years. This article will review such HBOC and PFC designs, including novel molecules which are still in development. We aim to identify and address the challenges in this field.

\section{POTENTIAL OF PFCs OR PERFLUROCHEMICALS AS A BLOOD SUBSTITUTE}

PFCs are long-chain polymers similar to Teflon. As they are biologically inert materials and 
dissolve 50 times more oxygen than plasma, they have a potential capacity to replace as a blood substitute, until other blood resources are available. Secondly, as they are made devoid of biological materials (potentially pathogenic in nature), the possibility of spreading infectious diseases via blood transfusion is eliminated.

However, there are challenges concerning PFCs. First, these molecules are insoluble in water and therefore they must be combined with emulsifiers. Adding emulsifiers can make them potentially dangerous. Secondly, they carry much less oxygen when compared to HBOCs. To counter this effect, more PFCs must be used which could again not be ideal.

\section{POTENTIAL OF HBOCS OR HEMOGLOBIN BASED OXYGEN CARRIERS AS A BLOOD SUBSTITUTE}

In ideal conditions, hemoglobin carries oxygen from the lungs to deliver to all body tissues. Herein, oxygen covalently binds to hemoglobin, and HBOCs share this natural mechanism of oxygen-binding hemoglobin. This property is unlike in the PFCs, and thus advantageous for HBOCs use. Secondly, as they are not contained in a cell membrane, the problem of blood typing is also eliminated.

However, the use of raw hemoglobin $(\mathrm{Hb})$ without modification cannot be replaced for blood because hemoglobin could actively break down into smaller peptide compounds which are toxic for blood $\mathrm{pH}$. Therefore, identifying a modified HBOC product, which remains stable and functional to replace blood's oxygencarrying capacity is researched upon. Currently, research is underway to stabilize Hemoglobin by modification, mostly by cross-linking molecules using different bonding partners, or molecules, so that Hemoglobin molecule remains intact without disintegrating or degrading into toxic molecules.

For example, methods employ the use of recombinant DNA technology, Polyethylene Glycol modified Liposome-Encapsulated Hemoglobin, nanoparticle and polymersome encapsulated hemoglobin, stabilized hemoglobin solutions, polymerized hemoglobin solutions, and conjugated hemoglobin solutions. Each of these is discussed below.

\section{TYPES OF HBOCS BEING DEVELOPED}

\section{A. Cell-Free Haemoglobin}

$\mathrm{Hb}$ obtained in its natural form can be sourced from human, bovine, salmon or recombinant bacteria. Free hemoglobin, which can also be referred to as naked hemoglobin, is the hemoglobin that is not in the Red Blood Cell. Since the hemoglobin is unbound and freed from its enclosure in the $\mathrm{RBC}$ which provides a 
range of antioxidant defenses, it is much more susceptible to being oxidized.

\section{IntramolecularlycrosslinkedHb}

Intramolecularly cross-linked $\mathrm{Hb}$ is the modified version of cell-free Hemoglobin which is cross-linked between subunits with different agents. It involves the cross-linking between two alpha and beta subunits, which reduces the affinity of hemoglobin for oxygen. For example, glycine, glutaraldehyde, O-raffinose, 3,5dibromosalicyl fumarate, pyridoxal-5-phosphate are used as linking agents. Many companies have ongoing projects and are on the verge of launching. The first being Baxter, which is working on modified Hemoglobin called HemAssist. Similarly, Hemopure (by Biopure), Optro (by Somatogen), Hemolink (by Hemosol) are being considered, as they differ only in the cross-linking agent.

\section{PEG-conjugated Hemoglobin}

PEGylation is a process in which polyethylene glycol polymer chains are covalently or noncovalently attached to molecules of macrostructures. When molecules undergo PEGylation, that is, with the introduction of strands of polyethylene glycol to the molecules, which are most typically proteins and antibody fragments, it has been shown to improve the safety and efficiency of drug treatments through increasing systemic retention. This improvement is achieved through altering physicochemical properties of the molecule such as conformation, electrostatic binding, and hydrophobicity. In the case of PEG-conjugated Hemoglobin, it is a type of surface conjugation on the cell-free Hemoglobin molecule by Maleimide-activated Polyethylene glycol or nick-named, Hb-Mal-PEG. It is being developed under the brand nameHemospan (by Sangart).

\section{Inter-molecularly cross-linked and polymer-tethered $\mathrm{Hb}$}

Another form of cell-free Hemoglobin is being developed by intra- and inter- molecularly cross-linked or tethered from polymer chains with the use of molecular agents like glutaraldehyde, poly-oxygen ethylene, Oraffinose. Different companies have called Polyheme (Northfield lab), pyridoxylatedHb, or PHP (Apex Biosciences), and others are also developing similar molecules.

\section{Polymerized Hemoglobin with Red Blood Cell-relevant redox enzymes}

Another cell-free hemoglobin being developed is inter-molecularly cross-linked or polymertethered in multiple copies and is packaged in association with enzymes like superoxide 
dismutase (SOD) and catalase (CAT). These molecules can maintain a redox environment for efficient Hemoglobin activity. It is a brand named Poly-Hb-SOD-CAT.

\section{B. Encapsulated Hb based HBOCs}

Unlike free or naked hemoglobin which is unbound and freed from its enclosure in the Red Blood Cell, encapsulated hemoglobin closely mimics hemoglobin's natural form in the human body. Hemoglobin in the human body is encapsulated within the red blood cell, providing it with anti-oxidant properties to ensure hemoglobin is circulated and delivered properly. Currently, scientists and researchers are working on numerous encapsulating technologies for Hemoglobin. The first being the usage of phospholipid vesicles or liposomes which are all biodegradable and much more biologically compatible within the human body.

\section{PLA-PEG Micro or nano-corpuscles}

PEG-PLA is also known as Poly (ethylene glycol)-Poly (lactic acid) block copolymer is used as a casing mechanism for hemoglobin to deliver oxygen and carbon dioxide. PEG-PLA has been prepared and characterized using the Fourier transform infrared spectrometer (FTIR). The results of using this method allowed for the formation of PLA-PEG-PolySFHb-SOD-CAT-
CA nanocapsules that can transport oxygen and carbon dioxide with increased antioxidant activity. These nano-capsule structures have a core-shell structure with a diameter of $100 \mathrm{~nm}$ which allows them to have a high entrapment efficiency, percent recovery, and good dispersion.

Other PLA-PEG nanocapsules have Hemoglobin encapsulated with Collodion (a nitrocellulose membrane), thus membranebound or PEG-PLA polymer (another membrane) creates a micro- or a nanoparticle. It is representative of an artificial Hemoglobin corpuscle.

\section{Polymer Shell Encapsulated Hemoglobin}

Polymer Shell Encapsulated Hemoglobin also known as PolymersomeEncapsulated Hemoglobin, is a new technology under development. Currently, it harvests bovine hemoglobin which is then encapsulated inside polymer vesicles (polymersomes) to form the polymer shell encapsulated Hemoglobin. The hemoglobin molecule being developed is encapsulated along with various redox enzymes (within Collodion or PEG-PLA based membrane); thus bound as a micro- or nanoparticle. It is also representative of an artificial Hemoglobin corpuscle. 


\section{VOLUME: 12, JULY-DECEMBER 2021}

\section{Liposome encapsulated Hemoglobin}

Liposome encapsulated hemoglobin is another HBOC that is the most promising encapsulated hemoglobin. They are currently being developed at the United States Naval Research Laboratory. Liposome encapsulated hemoglobin's biophysical properties closely mirror the properties of Red Blood Cells, which results in the oxygen-binding capabilities and affinity to be favorable. The hemoglobin molecule is encapsulated within sub-micron size lipid vesicles (or liposomes) and treated or PEGylated liposomes (Stealth liposomes).

It's representative of Liposome Encapsulated Hemoglobin (LEH). Other names used by different companies are TRM-645 Neo Red Cells, Hemoglobin vesicles, or HBV, etc.

\section{Polymersome Encapsulated $\mathrm{Hb}$}

Polymersome encapsulated hemoglobin is selfassembled from various materials that are biodegradable and biocompatible making the amphiphilic block of copolymers: PEG-PBD, PEG-PLA, PEG-PCL. Specifically, poly (ethylene oxide) (PEO), poly (caprolactone) (PCL), and poly (lactide) (PLA). The hemoglobin is encapsulated within a sub-micron size polymer vesicle (polymersome). The encapsulating method is currently a popular method for protein carriers, especially hemoglobin-based oxygen carriers. While being tested in hamsters experiencing hemorrhagic shock, the encapsulated hemoglobin was able to reach loading capacities that were comparable to literature values that supported the successful resuscitation. Consequently, the Polymersome encapsulated hemoglobin shows much promise as a successful oxygen carrier in the future.

\section{Novel molecules and designs for HBOCs}

\section{HemoTech (HemoBioTech)}

The design that has been pioneered by Dallasbased companyHemoBioTech, has proven to be an effective oxygen carrier in treating blood loss, anemia, and ischemic vascular conditions. It has purified bovine hemoglobin that is intramolecularly cross-linked with ATP; intermolecularly cross-linked with adenosine, and further conjugated with GSH or reduced glutathione. HemoTech uses ATP, adenosine, and GSH, which are all classified as pharmacologically active molecules as chemical modifiers. The ATP molecule ensures vascular tone through purinergic receptors, whereas the adenosine stimulates adenosine receptors. They together counteract the vasoconstrictive properties of hemoglobin and finally, the GSH protects the heme group from various oxygenrelated molecules like $\mathrm{CO}$ and NO. Hemotech 
VOLUME: 12, JULY-DECEMBER 2021

has advanced to Phase 1 of clinical trials.

\section{HEMOXYCarrier (Hemarina)}

Hemarina is a revolutionary company founded in 2007 by Frank Zal that harnesses the power of a natural biopolymer resulting from 450 million years of evolution - obtained from a marine annelid. Hemarina technology uses this natural biopolymer. It is based on natural extracellular hemoglobin that has a high molecular weight, allowing it to function at a large temperature range $\left(4^{\circ} \mathrm{C}\right.$ to $\left.37^{\circ} \mathrm{C}\right)$. It uses giant extracellular hemoglobin $(3600 \mathrm{kDa})$. Furthermore, it does not require a cofactor to release oxygen. Hemarina claims that an essential advantage of this technology is the absence of a vasoconstrictor and hypertensive effect. Hemarina's technologies have several promising applications, including HEMOXYCarrier, a universal therapeutic oxygen carrier. HEMOXYCarrier is currently undergoing preclinical studies, showing promising results in terms of showing no significant impact on mean arterial blood pressure in comparison to other HBOCs that use bovine or human hemoglobin.

\section{Core-Shell Bovine Hemoglobin and}

\section{Human HSA}

Recently, core-shell small clusters of protein made from bovine hemoglobin and human serum albumin (HSA) were used to create $\mathrm{Hb}$ -

HAS. This was done through the conjugation of the human serum albumin on $\mathrm{Hb}$, accomplished via the linkage of $\mathrm{Hb}$ surface lysines to HSA cysteine-34 using alpha-succinimidyl-epsilonmaleimide-cross-linker. The benefit of this potential oxygen carrier is that these $\mathrm{Hb}-\mathrm{HSA}$ clusters have, in preclinical trials, been reported to lower the risks of rapid clearance and extravasation. Consequently, this has improved high circulation stability and residence time.

An extension of this oxygen carrier has recently also been developed. A further modification of these Hb-HSA core-shell nanoclusters was pioneered to protect the $\mathrm{Hb}$. Antioxidant enzymes and platinum nanoparticles were introduced into the HSA pockets to achieve $\mathrm{Hb}$ protection. Although these nano-cluster designs have only been tested in vitro, they display positive results.

\section{Template induced layer-by-layer (L-B-L) assembly}

In this case, there is template-induced layer-bylayer (L-B-L) assembly of cationic Hemoglobin. It features a covalent L-B-L assembly formed with the aid of anionic polymers such as Dialdehyde heparin (DHP). DHP is an integral part of the L-B-L assembly, used as a crosslinker and wall component without the usage of extraneous or toxic crosslinking agents. This 
step is followed by the dissolution of the template core. The template-induced layer-bylayer assembly's oxygen-carrying capacity is shown through the conversion of the deoxy- $\mathrm{Hb}$ state of the microcapsules into the oxy-Hb state. The quintessential result behind this is that the $\mathrm{Hb}$ L-B-L assembly demonstrates oxygencarrying properties, biocompatibility, and hemocompatibility, suggesting the viability of the L-B-L assembly as a blood substitute.

\section{Direct conjugates of $\mathrm{Hb}$ on the hydrophobic block}

In this case, hemoglobin was covalently conjugated directly to the hydrophobic or hydrophilic domain of block-copolymers. Then they are subsequently processed to the micellization of polymer molecules to form $\mathrm{Hb}$ encapsulated micelle nanoparticles. There have been two research papers that have investigated the synthesis of hemoglobin conjugates using micelles. Both use amphiphilictriblock copolymers.

The first by $\mathrm{Li}$ et al., uses these amphiphilictriblock copolymers with carbonyl groups. Condensation reactions are then performed between the carbonyl and amino groups of $\mathrm{Hb}$ to produce conjugated $\mathrm{Hb}$ on the copolymer micelles. This process ensures that the $\mathrm{Hb}$ retains its gas-binding capabilities.
Furthermore, when the $\mathrm{pH}$ is adjusted closer to the isoelectric point of $\mathrm{Hb}$, it allows for the $\mathrm{Hb}$ to be rearranged. Essentially, this innovation could perhaps light the path to a new method in the creation of protein carriers that is different from the traditional method of encapsulation.

The second paper by Qi et al. uses the amphiphilictriblock copolymers that are selfassembled into core-shell micelles in an aqueous solution. Next, a thiol-Michael reaction (an addition reaction) is performed to yield thiolated hemoglobin that is conjugated with micelles, forming hemoglobin nanoparticles (HbNs) about $200 \mathrm{~nm}$ in diameter. This $\mathrm{Hb}$ has been tested in vitro, showing no large signs of negative effects on blood components. Like the amphiphilictriblock copolymer with carbonyl groups method of conjugating hemoglobin, this method also is displaying potential as an oxygen carrier or even as a protein carrier.

\section{6. $\mathrm{Hb}$ co-precipitated with $\mathrm{CaCO}$ or $\mathrm{MnCO3}$}

In this case, hemoglobin is co-precipitated with $\mathrm{CaCO} 3$ or $\mathrm{MnCO} 3$. To achieve this, hemoglobin is loaded in microparticles through coprecipitation with $\mathrm{CaCO} 3$, followed by stabilization through cross-linking (e.g. with glutaraldehyde) and further complexed with anionic proteins like HSA to form nano- or 


\section{VOLUME: 12, JULY-DECEMBER 2021}

micro-scale clustered particles. The quantity of hemoglobin per microparticle closely resembles that of natural RBCs. In studies, similarities in the oxygen equilibrium kinetics between free $\mathrm{Hb}$ and the $\mathrm{Hb}$-microparticles have been drawn. Additionally, the analogous Hbmicroparticles were able to carry $80 \%$ of the oxygen in comparison to natural RBCs. To ensure the biocompatibility of these microparticles, they have been modified using polyethylene glycol to protect and stabilize the system. With the usage of the $\mathrm{CaCO} 3$ particle, the hemoglobin spheres have great potential to continue development as an oxygen-carrying substitute for blood.

\section{Mechano-biologic mimicry of RBCs}

In this case, there is a direct mechano-biological mimicry of RBCs. Since it has been assumed that the RBCs elastic modulus is the property that is responsible for the cell's circulation time and bio-distribution, a group of scientists created microparticles with tunable elasticity to mimic RBCs. Essentially the $\mathrm{Hb}$ was encapsulated within the RBC morphologymimetic flexible discord polymer-based micro polymer. This membranous structure is formed through lithographic or template-inducing printing techniques. Despite trying to accurately mimic the mechanobiological functions of RBCs, the alterations made to the RBCs bypassed several organs, including the lungs, but were able to demonstrate circulation times longer than traditional microparticles.

\section{Erythromer (Nanocrit):}

Erythromer is currently being developed by the company- KaloCyte. The molecule of Erythromer is a dried, bio-inspired artificial red blood cell. KaloCyte envisions Erythromer as useful when red blood cells are unavailable, undesirable, or in short supply. This product has proven to be stable for months even when freeze-dried, allowing for stockpiling to be possible, ideally for use in emergencies. This product is a red blood-like powered, as it is derived from the hemoglobin of humans. Modifications are made by coating it with a synthetic polymer: allowing the microparticle to sense $\mathrm{pH}$ changes. Consequently, for it to pick up oxygen in $\mathrm{pH}$-high areas, and dispose of in areas where the levels are low becomes automatic. The hydrophobic tails are conjugated with amphiphilic polythene imine (PEI) which are already modified with palmitic acid and are called PEI molecules. They self-assemble with recombinant Hemoglobin, DPG, and antioxidants in reverse. On self-assembly, they may also form micelles (called nanobialys) that can encapsulate $\mathrm{Hb}$, and maintain a redox enzymatic environment for $\mathrm{Hb}$ activity by sequestering 2, 3 DPG, and leuko-methylene blue in the micelle particle. As of now, trials 
have been successful in rats, mice, and rabbits, with human trials being planned.

\section{HBOC'S BENEFITS}

Hemoglobin based oxygen carriers (HBOCs) are a possible method to use for formulating artificial blood. Because HBOC's lack membranes, there is no need for patients to be cross-matched with a specific type of blood. HBOC's can be given to anyone who requires blood as it does not require blood typing to be performed. This can have numerous clinical benefits, especially in emergency settings. In comparison to PFC's, HBOC's carry more oxygen rendering them more oxygenated than PFC's. There is a great variety of HBOC's that currently exist and are still in development. These HBOC's each have their benefits and drawbacks. As there is no clear substitute for blood each of the HBOC's is a potential replacement to blood.

\section{SURFACE MODIFIED HEMOGLOBIN}

Surface-modified hemoglobin is developed through the attachment of large molecules such as polyethylene glycol to surface lysine groups essentially increasing the viscosity and oncotic pressure of the substance. HBOC's in blood would immediately be targeted by the body's immune system, and to avoid this, there is the surface modification of hemoglobin. This protects to ensure that the body's immune system does not attack it. This ensures a greater circulation time after administration. Due to the surface modification that ensures the protection against the immune system, a major benefit of surface-modified hemoglobin is that they have a lower incidence of antibody production resulting in the patient's body being able to readily accept it.

Although surface-modified hemoglobin is still in the development phase, many companies are experimenting with molecules that are of various sizes, each of which has its benefits. Enzon is developing a smaller-sized hemoglobin molecule that allows it to treat diseases such as stroke and cancer. The small size of the molecule allows it to reach areas in which erythrocytes are unable to reach. An example of this is that in Cancer, these HBOC's can carry oxygen to tumor cells, to increase their susceptibility to chemotherapy or radiation treatments.

\section{LIPOSOME}

ENCAPSULATED

\section{HEMOGLOBIN}

Another HBOC is liposome-encapsulated hemoglobin. This type of HBOC is a lowtoxicity and spatially isolated HBOC. Liposome encapsulated hemoglobin is primarily compatible with the immune system as it has a negatively charged lipid that limits aggression and unlike surface-modified hemoglobin, it is more suitable to be used in an emergency trauma setting. Additionally, liposome- 
encapsulated hemoglobin (hemosomes) allows for greater manipulation that can allow scientists to alter multiple physicochemical properties and circulation lifetime. Firstly, the P50 of modified hemoglobin can be altered by the modification of liposomes for easier oxygen unloading. Additionally, the oxygen affinity can be adjusted by co-encapsulating an allosteric effector such as a pyridoxal 5' phosphate. Further modification with polyethylene glycol increases the half-life of the HBOC, makes it water-soluble, lowers antigenicity, and increases the specific site targeting.

\section{HBOC'S DRAWBACKS}

HBOC's require intramolecular cross-linking between two alpha and beta subunits which can reduce the affinity of hemoglobin for oxygen. Additionally, the HBOC's circulation half-life is shorter than RBC's, meaning that HBOC's need to be replaced at a higher rate than RBC's which can become expensive and inefficient. Another drawback of HBOC's is that, unlike RBCs, hemoglobin and other plasma proteins can exert some osmotic pressure.

Consequently, cellular hemoglobin can alter the intravascular volume of blood and act as a plasma expander which can be deadly during circulation. Also during circulation, HBOC's release free radicals in the body from free hemoglobin and break down products like heme and iron. As heme and iron are compulsory for gas exchange to occur, the breakdown of these molecules can have deadly consequences. Finally, another drawback of HBOC's is that the first choice for obtaining hemoglobin is blood which is in limited supply. Consequently, bovine blood must be used but this can harbor pathogens responsible for causing bovine spongiform encephalopathy (Creutzfeldt-Jakob disease). The only other feasible solution to avoid causing spongiform encephalopathy is using Recombinant Hemoglobin processes (E.coli). However, the extremely high costs of this technique are a major hindrance to the development of HBOC's.

\section{PFC'S BENEFITS}

Perfluorocarbons have revealed themselves as clinically useful oxygen carriers. They are capable of carrying oxygen and carbon dioxide without binding, which means that it does not react with oxygen or any other gases. This is extremely beneficial as it allows PFC's to be used as a closer substitute to blood. PFC's also increase the solubility of oxygen in the plasma which means it is better absorbed by the blood and can be better used by the organs in the body. Furthermore, it allows for the easier and faster transfer of oxygen from cells to tissues. PFC's do not have any effect on temperature, $\mathrm{pH}$, and 2,3 diphoglycerate on the dissolution of oxygen. Although PFC's are still in their development stage, first-generation PFC's such 


\section{VOLUME: 12, JULY-DECEMBER 2021}

as Fluosol have been approved by the FDA as a blood substitute for cardiac surgery. Secondgeneration PFC's are still in development, but their oxygen-carrying capacity is substantially higher than first-generation and their excretion of gas is faster but at the cost of lower tissue retention.

\section{PFC'S DRAWBACKS}

Despite the promising clinical results of PFC's, the overall development process of PFC's has been quite slow due to the property that they are immiscible in aqueous solutions. Since blood is an aqueous solution, PFC's have struggled to form homogeneous solutions with blood. Consequently, they need to be emulsified with the addition of lipids. Since the size of PFC's is approximately 100 times smaller than RBC's, this hinders their oxygen-carrying capacity. At times, PFC has been known to cause flu-like symptoms which occur due to opsonization and phagocytosis of PFC emulsion by the recipient organism's immune system. Additionally, since PFC's have a high affinity for oxygen, exposures to high oxygen concentration during PFC infusion can result in oxygen toxicity. The usage of PFC can overload the reticuloendothelial system, thereby suppressing its function. Additionally, a drawback to PFC's is that PFC products cannot be used by the human body as they need to be removed every 18-24months, in addition causing a higher rate of neurological complications having been found in human cardiac cases. 


\section{VOLUME: 12, JULY-DECEMBER 2021}

\section{REFERENCES}

[1] Sarkar Suman. Artificial blood. Indian J Crit Care Med. 2008 Jul-Sep; 12(3): 140-144. doi: 10.4103/0972-5229.43685. PMCID: PMC2738310

[2] RudrashishHaldar, Devendra Gupta, Shweta Chitranshi, Manish Kumar Singh, SumitSachan. Artificial Blood: A Futuristic Dimension of Modern Day Transfusion Sciences. CardiovascHematol Agents Med Chem. 2019 May; 17(1): 11-16. Published online 2019 May. doi: 10.2174/1871525717666190617120045 PMCID: PMC6864588

[3] Samira Moradi, Ali Jahanian-Najafabadi, MehryarHabibiRoudkenar. Artificial Blood Substitutes: First Steps on the Long Route to Clinical Utility. Clin Med Insights Blood Disord. 2016; 9: 33-41. Published online 2016 Oct 27. doi: 10.4137/CMBD.S38461. PMCID: PMC5084831

[4] Keipert PE. Hemoglobin-Based Oxygen Carrier (HBOC) Development in Trauma: Previous Regulatory Challenges, Lessons Learned, and a Path Forward. AdvExp Med Biol. 2017;977:343-350. doi: 10.1007/978-3-319-55231-6_45. PMID: 28685464.

[5] Schaer DJ, Buehler PW, Alayash AI, Belcher JD, Vercellotti GM. Hemolysis and free hemoglobin revisited: exploring hemoglobin and hemin scavengers as a novel class of therapeutic proteins. Blood. 2013;121(8):1276-1284. doi:10.1182/blood-2012-11-451229

[6] Rudolph AS. Encapsulated hemoglobin: current issues and future goals. Artif Cells Blood SubstitImmobilBiotechnol. 1994;22(2):347-60. doi: 10.3109/10731199409117425. PMID: 8087251.

[7] Ghasemi, R., Abdollahi, M., EmamgholiZadeh, E. et al. mPEG-PLA and PLA-PEG-PLA nanoparticles as new carriers for delivery of recombinant human Growth Hormone (rhGH). Sci Rep 8, 9854 (2018).

[8] Dian RA, Andre FP. Polymersome Encapsulated Hemoglobin: A Novel Type of Oxygen Carrier. Biomacromolecules 2005, 6, 4, 2172-2181 Publication Date:June 4, 2005

[9] Rameez S, Alosta H, Palmer AF. Biocompatible and biodegradable polymersome encapsulated hemoglobin: a potential oxygen carrier. BioconjugChem 2008 May;19(5):1025-32. Epub 2008 Apr 29. PMID: 18442283.Dian R. Arifin and Andre F. Palmer. Polymersome Encapsulated Hemoglobin: 


\section{VOLUME: 12, JULY-DECEMBER 2021}

A Novel Type of Oxygen Carrier. Biomacromolecules 2005 6(4), 2172-2181. DOI: $10.1021 / \mathrm{bm} 0501454$

[10] Farmer MC, Johnson SA, Beissinger RL, Gossage JL, Lynn AB, Carter KA. Liposomeencapsulated hemoglobin: a synthetic red cell. AdvExp Med Biol. 1988;238:161-70. doi: 10.1007/978-1-4684-7908-9_13. PMID: 3250239.

[11] Sarkar, Suman. “Artificial blood.” Indian journal of critical care medicine: peer-reviewed, official publication of Indian Society of Critical Care Medicine vol. 12,3 (2008): 140-4. doi:10.4103/0972-5229.43685.

[12] Surface Modification of Hemoglobin-Based Oxygen Carriers Reduces Recognition by Haptoglobin, Immunoglobulin, and Hemoglobin Antibodies 